\title{
The basic tastants in aversion conditioning: Evidence for sensory preconditioning and not potentiation
}

\author{
GREGORY J. PRIVITERA and ELIZABETH D. CAPALDI \\ State University of New York, Buffalo, New York
}

\begin{abstract}
In Experiment 1, a potentiation paradigm was used to test the relative influence of odor and taste with two 2 basic tastants (i.e., salt and sweet) in conditioned aversion learning. Experiment 1 showed that aversions to tastants (salt or sweet presented in a manner by which it could be tasted) were established only in subjects trained with the tastant, not the odor (i.e., salt or sweet presented in a manner by which it could not be tasted). Experiment 2 demonstrated, with a sensory preconditioning procedure, that the expression of an aversion to tastants was dependent on previous tastant experience prior to odor aversion training. These results suggest that while subjects can smell salt and sweet solutions, these odors are neither sufficient nor necessary for the expression of a conditioned tastant aversion.
\end{abstract}

Some research suggests that stimuli typically referred to as the basic tastes (i.e., sweet, sour, salt, and bitter) can be detected by olfaction (Benjamin, 1960; Rhinehart-Doty, Schumm, Smith, \& Smith, 1994) and, therefore, should more appropriately be termed basic tastant stimuli. A tastant, or basic tastant, is defined as a sweet, sour, salty, or bitter solution presented in a manner by which it can be tasted. For example, a tastant presented in a bottle from which subjects can drink allows them to ingest the solution and, therefore, be exposed to both the taste and the potential odor components of that solution. With tastant stimuli, it has been reported that rats can detect solutions such as sucrose (Rhinehart-Doty et al., 1994) and quinine (Benjamin, 1960; Smith \& Theodore, 1984) prior to ingestion. Henkin (1962) showed that humans could also detect what are typically termed tastants, such as sodium chloride $(\mathrm{NaCl})$, potassium chloride $(\mathrm{KCl})$, hydrochloride, urea, and sucrose, without ingesting these solutions. Therefore, it is assumed that subjects can smell and taste these solutions when they are consumed.

Also, in the present investigations, an odor is defined as being limited to a sweet, sour, salty, or bitter solution presented in a manner by which it cannot be tasted. For example, a solution can be injected into a pad on a disk presented outside the rats' cage and placed under a tube containing distilled water from which the rats can drink. When presented in this manner, subjects not only smell these stimuli, but also can express aversions to these odors (Capaldi, Hunter, \& Privitera, 2004). Capaldi and colleagues trained rats with saccharin (sweet) and $\mathrm{NaCl}$ (salt) as conditioned stimuli and presented the stimuli either in bottles as tastants or on disks

Correspondence concerning this article should be addressed to G. J. Privitera, Department of Psychology, Arizona State University, 950 S. McAllister Street, Tempe, AZ 85287 (e-mail: gjp@asu.edu). located outside the cage front as odor cues. In test, the rats expressed aversions to the tastants and the odors.

In a subsequent experiment (Capaldi et al., 2004, Experiment 2), some rats were made anosmic following training in order to determine whether the odor stimuli were truly detected by olfaction when they were presented on disks. The results showed that disrupting olfaction in rats eliminated the expression of an odor aversion in rats trained and tested with solutions presented as odors on disks. Thus, the tastants had a conditionable odor component, since disrupting olfaction in rats eliminated the conditioned odor aversion. In addition, disrupting olfaction had no effect on the expression of a conditioned aversion to the tastants in rats trained and tested with tastants. Thus, olfactory impaired and intact rats expressed statistically similar aversions to tastants, suggesting that although subjects can smell salt and sweet solutions, these odors may not significantly contribute to a conditioned tastant aversion. On the basis of the results of Capaldi et al., it is presently unclear how much influence odor may have on the expression of a conditioned aversion to tastants. Using saccharin and salt as conditioned cues, the present experiments were designed to specifically investigate the influence odor detection has on conditioning to these stimuli.

\section{EXPERIMENT 1}

In Experiment 1, we investigated two main hypotheses, using saccharin and $\mathrm{NaCl}$ as conditioned cues. First, we wanted to determine whether a tastant could potentiate an odor aversion. Second, we investigated whether subjects would generalize an odor aversion to the tastant following odor aversion training. The results from this experiment can be used to infer the differential influence each component has on the expression of a conditioned aversion to the tastant. 


\section{Method}

Subjects. The subjects were 48 experimentally naive, male Sprague Dawley rats from Harlan Co., Indianapolis. Upon arrival, they were 60 days old and weighed from 275 to $300 \mathrm{~g}$. At the start of the experiment, they were 70 days old.

Materials and Apparatus. The solutions were presented in 50-ml Nalgene tubes with rubber stoppers and metal spouts. The stimuli used in training and test were $0.2 \% \mathrm{NaCl}$ (Fisher Scientific, Fair Lawn, NJ; wt $/ \mathrm{vol}$ ) and $0.15 \%$ saccharin (Sigma Chemical, St. Louis; $\mathrm{wt} / \mathrm{vol}$ ). These stimuli were mixed in distilled water and were presented either in bottles for ingestion (i.e., tastant) or on disks that were attached to the metal spouts of tubes containing distilled water (i.e., odor). The disks were weigh dishes $(3.8 \times 2.54 \mathrm{~cm}$ polystyrene; Fisher Scientific) that contained a cotton pad. The $\mathrm{NaCl}$ or saccharin solution $(2 \mathrm{ml})$ was injected onto this pad immediately prior to the presentation of a solution. The tip of the metal spout of a bottle containing distilled water entered the cage, and the disks sat directly behind the tip on the outside of the cage. An aluminum screen was placed directly in front of the disk to prevent the rats from interfering with them. When the animal licked the tube's spout, its nose came within $2 \mathrm{~cm}$ of the disk. In training, the stimuli were paired with an injection of either $0.15 \mathrm{M} \mathrm{LiCl}$ (Fisher Scientific; $\mathrm{wt} / \mathrm{vol}$ ) or $0.9 \%$ saline. All training and testing was conducted in the rats' home cages. A 12:12-h light:dark cycle was always in effect, with lights on at $6 \mathrm{a} . \mathrm{m}$. The rats were run during the light portion of the light:dark cycle.

Procedure. On Day 1, all water bottles were removed from the cages at 8:00 a.m. On Days 2-11, all the rats received restricted access to water for $30 \mathrm{~min}$ at 3:00 p.m., and all chow (PMI Feeds, St. Louis) was removed $1 \mathrm{~h}$ prior to water access and was replaced $1 \mathrm{~h}$ later. In training and test, water access $(30 \mathrm{~min})$ was given $1 \mathrm{~h}$ following the completion of the experimental procedures. Chow was removed $1 \mathrm{~h}$ prior to the experimental procedures and was replaced $1 \mathrm{~h}$ following water access each day during training and test.

Training began on Day 12. During training, there were 4 conditioning days alternating with 4 rest days. The solutions were presented on an ABBA schedule over days. The $\mathrm{NaCl}$ solutions were presented on A days, and the saccharin solutions were presented on B days. On conditioning days, all the consumed solutions (distilled water, $\mathrm{NaCl}$, or saccharin) were presented in $40-\mathrm{ml}$ portions for $10 \mathrm{~min}$ each day. On rest days, each rat received 30 -min access to water. On the conditioning days, half the rats $(n=24)$ received one solution, followed immediately by an intraperitoneal (i.p.) injection of lithium chloride $(10 \mathrm{ml} / \mathrm{kg}, \mathrm{LiCl})$. The other half of the rats received the same solution, followed immediately by an i.p. injection of saline $(10 \mathrm{ml} / \mathrm{kg})$. The stimuli were counterbalanced, so that for half the rats in each group $\mathrm{LiCl}$ followed $\mathrm{NaCl}$ and saline followed saccharin. The remaining half of rats in each group received the reverse pairings over days. For half the rats, the stimuli were presented in bottles for ingestion during training (i.e., tastants). For the other half of the rats, the stimuli were presented on disks outside the cage front during training (i.e., odor), so that the subjects consumed distilled water from the bottles and, presumably, could smell the salt and sweet solutions presented on these disks.

Following training, the subjects were divided into four groups (12 subjects in each group): two groups for which the presentation of stimuli during training and test differed (Groups Tastant-Odor and Odor-Tastant) and two groups for which the presentation of stimuli during training and test were the same (Groups Tastant-Tastant and Odor-Odor). The subjects receiving a tastant during training were divided into the tastant groups (Groups Tastant-Odor and TastantTastant) and were matched so that each group of rats acquired similar aversions in training. The subjects were matched on the basis of the amount consumed during the second trial of conditioning. The subjects receiving an odor during training were divided into the odor groups (Groups Odor-Tastant and Odor-Odor) and were also matched so that each group of rats acquired similar aversions in training.
There were 4 days of testing immediately following training. All the groups received a two-bottle test (side by side) for $15 \mathrm{~min}$. For Group Tastant-Odor and Group Odor-Odor, the two-bottle test was between two bottles of distilled water with either $\mathrm{NaCl}$ or saccharin presented on a disk attached to the distilled water tube. For Group Odor-Tastant and Group Tastant-Tastant the two-bottle test was between $\mathrm{NaCl}$ and saccharin presented in bottles for ingestion. Placement of the tubes (on either the left or the right) in the cages was on an ABBA schedule. For all of the rats, during testing, one tube was placed into each cage and moved briefly so that each rat would approach and contact the tube; then the same was done for the other tube to ensure that the rats contacted both tubes.

Data analyses. In training, an ANOVA included counterbalancing (evaluating any effects of the particular stimulus that was paired with $0.9 \%$ saline or $0.15 \mathrm{M} \mathrm{LiCl})$ as the between-subjects factor, and days and pairing $(0.9 \%$ saline-paired solution vs. the $0.15 \mathrm{M} \mathrm{LiCl-paired}$ solution) were included as the within-subjects factors. Groups were included as an additional between-subjects factor for testing.

\section{Results and Discussion}

In training, the term aversion referred to reduced consumption of the tastant or distilled water associated with the odor cue paired with $\mathrm{LiCl}$ from Day 1 to Day 2 of training. All the groups acquired an aversion to the tastant or odor cue in training $[F(1,40)=39.84, p<.001$; see Figure 1]. Also, whereas consumption of the LiCl-paired solutions decreased from Day 1 to Day 2 of training, overall consumption remained the same for all the groups $[F(3,44)=0.36, p>.75]$.

In test, the term aversion referred to reduced consumption of the tastant or distilled water associated with the odor cue paired with $\mathrm{LiCl}$, in comparison with the stimulus paired with saline. Although the aversion extinguished from the first to the last day of test $[F(1,40)=22.53, p<$ $.001]$, the overall aversion was significant, as indicated by the effect of pairing $[F(1,40)=208.90, p<.001]$, and this effect varied by group $[F(3,40)=28.74, p<.001$; see Figure 2]. As is shown in Figure 2, only Group OdorTastant failed to express an aversion in test. NewmanKeuls tests confirmed that all the groups, except Group Odor-Tastant, expressed a significant aversion $(p<$

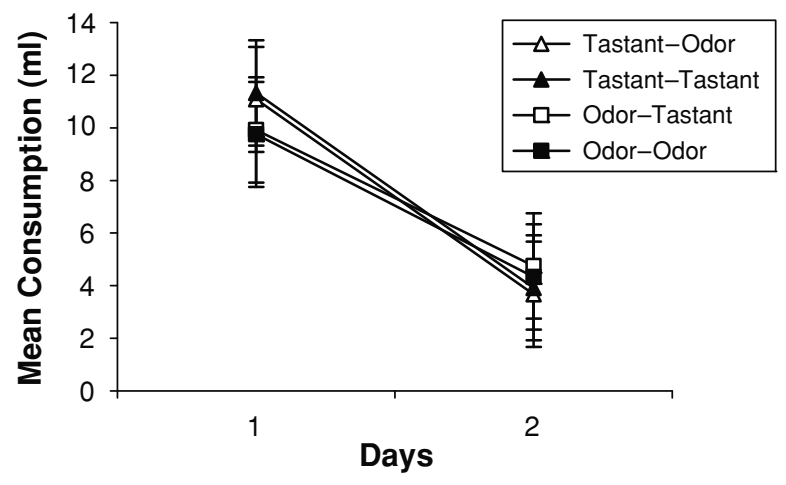

Figure 1. For each group in Experiment 1, mean consumption (in milliliters) during training of the solution that had been paired with $0.15 \mathrm{M} \mathrm{LiCl}$ over days. For clarity, consumption of solutions paired with $0.9 \%$ saline is not included, nor did consumption of these solutions differ significantly over days. Vertical lines represent the standard error of the mean. 


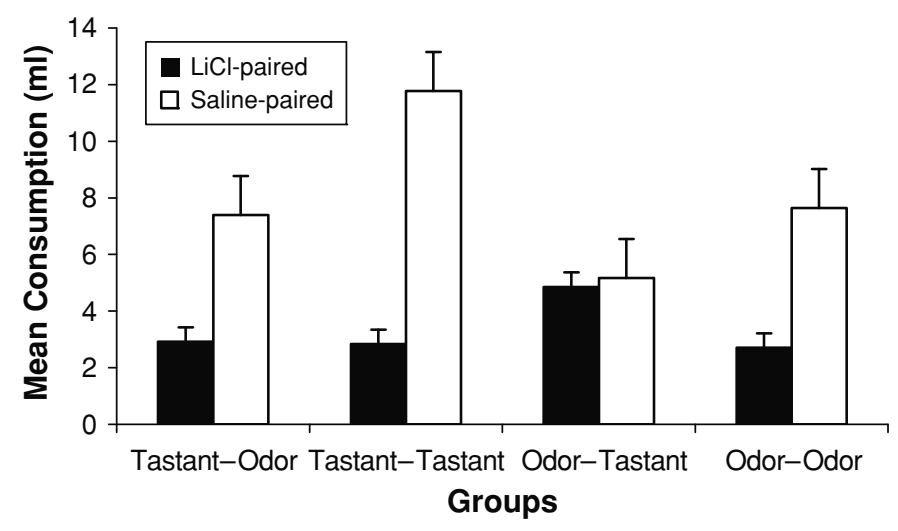

Figure 2. For each group in Experiment 1, mean consumption (in milliliters) pooled across the four test trials of the solution that had been paired with $0.9 \%$ saline versus the solution that had been paired with $0.15 \mathrm{M} \mathrm{LiCl}$ in training. Vertical lines represent the standard error of the mean.

$.01)$ and the aversion was significantly larger for Group Tastant-Tastant than for any other group $(p<.03)$.

Notably, Group Tastant-Odor and Group Odor-Odor expressed statistically similar aversions in test $(p>.05)$. These results demonstrate that odor aversions to saccharin and $\mathrm{NaCl}$ were similarly expressed in test, regardless of whether the subjects were conditioned with tastants (as in Group Tastant-Odor) or odors (as in Group Odor-Odor). Thus, pairing a tastant with $\mathrm{LiCl}$ (Group Tastant-Odor) resulted in the expression of an odor aversion of the same strength as the odor aversion expressed following odoralone conditioning (Group Odor-Odor). Hence, this experiment showed no evidence for cue potentiation or overshadowing, in that conditioning with the tastant failed to interfere with (overshadowing) or enhance (potentiation) the expression of an odor aversion in test. One explanation for this finding may be that the tastant cue simply generalizes to the odor component in test and does not act to facilitate (or interfere with) conditioning to the odor during aversion conditioning trials.

Also, the finding that Group Odor-Tastant, which was trained with an odor and tested with a tastant, failed to express an aversion to the tastant in test, suggests that the subjects were unable to recognize the tastant as a conditioned cue in test. One main concern requires an explanation for why tastant conditioning generalizes to the odor in test for Group Tastant-Odor, but odor conditioning does not generalize to the tastant (which includes the odor component) in test for Group Odor-Tastant. As has been indicated by some reports on potentiation (Ellins, Cramer, \& Whitmore, 1985; Ellins \& von Kluge, 1987), it is possible that without prior ingestion of a basic tastant during training, the odor component may not be recognized as an attribute of that basic tastant and, thus, may not generalize to the tastant in test. The subjects in Group Odor-Tastant were trained with an odor and then tested for an aversion to the tastant (i.e., trained with a saccharin or $\mathrm{NaCl}$ solution presented on a disk outside the cage front, then tested with the saccharin or $\mathrm{NaCl}$ solution presented in a bottle for ingestion). Given that these subjects did not express an aversion, this may suggest that although subjects can express aversions to sweet and salt solutions without ingesting them, this odor component may not be inherently recognized as a component deriving from a basic tastant. This would mean that Group Odor-Tastant was, in fact, the only group to experience a novel component of the basic tastant stimulus in test (i.e., the subjects had never tasted the solution prior to test). The present findings confirm this assertion by showing that, as would be expected (Domjan, 1976), the presence of this relatively novel component during testing reduced overall consumption in test $[F(3,40)=19.29, p<.001]$ and also interfered with the expression of an aversion to the tastant in this group.

One interpretation for this finding would be to assert that in order for a tastant to be recognized by olfaction, subjects must have prior experience with the tastant for the odor to come to be associated with and generalize to the tastant following aversion training. To test this, we used a sensory preconditioning (SPC) paradigm (Brogden, 1939) in Experiment 2, to determine whether preexposure to the tastant solution would be sufficient to facilitate the expression of an aversion to the tastant following training with an odor.

\section{EXPERIMENT 2}

The stimuli used in Experiment 2 were the same as those used in Experiment 1. The main procedural change in Experiment 2 was that two groups received preexposure to the tastant (the preexposure groups) and the remaining two groups did not (the control groups) prior to training with the odor cue. All the subjects received odor aversion training.

\section{Method}

Subjects $(n=48)$, of the same description as those in Experiment 1 , received the same access to water and chow as that described in Experiment 1 prior to and during the experiment. There 
were 4 days of preexposure, which began on Day 12. Access to water and chow during this phase was the same as that described for training and test in Experiment 1. The rats were divided into four groups (12 subjects in each group): Two groups received preexposure to tastants prior to training (Groups PRE-Odor-Tastant and PRE-Odor-Odor); the two remaining groups did not (Groups CNT-Odor-Tastant and CNT-Odor-Odor). Among the preexposed groups, $\mathrm{NaCl}$ and saccharin were presented on an $\mathrm{ABBA}(\mathrm{A}=$ $\mathrm{NaCl}, \mathrm{B}=$ saccharin) schedule for 10 min each day. The nonpreexposed groups (i.e., the CNT groups) were presented with distilled water on each of these days.

Training began on Day 16. All the training procedures were the same as those described in Experiment 1 for the groups trained with an odor (i.e., Group Odor-Odor and Group Odor-Tastant in Experiment 1). Thus, all the subjects received odor aversion training with $\mathrm{NaCl}$ and saccharin presented as odor cues on disks attached to the metal spouts of tubes containing distilled water, from which they drank.

There were 4 days of testing immediately following training conducted as described in Experiment 1. Half the rats were tested with odor cues (i.e., Group PRE-Odor-Odor and Group CNT-OdorOdor); the other half were tested by consumption of the tastant (i.e., Group PRE-Odor-Tastant and Group CNT-Odor-Tastant).

\section{Results and Discussion}

The data analyses were the same as those used in Experiment 1, except that groups was included as a factor for training and pairing was deleted as a factor for preexposure. All the groups consumed similar amounts of solution during the preexposure phase, with no evidence of group differences in consumption $(p>.05)$. Mean consumption of the tastants during the preexposure phase was 11.11 (PRE-Odor-Odor), and 10.98 (PRE-OdorTastant). Mean consumption of distilled water was 9.10 (CNT-Odor-Odor), and 8.79 (CNT-Odor-Tastant).

In training, all the groups acquired an aversion to the distilled water associated with the LiCl-paired odor cue $[F(1,40)=114.07, p<.001]$, and this did not vary by group $(p>.20$; see Figure 3$)$. Consumption of the salinepaired solutions did not vary by day or group $(p>.40)$. Also, although consumption decreased from Day 1 to Day 2 of training, overall consumption of LiCl-paired and saline-paired solutions did not significantly vary in training $(p>.05)$.

In testing, all the groups except Group CNT-OdorTastant $(p>.05)$ expressed a significant aversion to the LiCl-paired solution $[F(1,40)=168.23, p<.001]$. As can be seen in Figure 4, only Group CNT-Odor-Tastant failed to express an aversion in test. This was confirmed in the ANOVA, which showed that the aversion varied by group $[F(3,40)=16.56, p<.001]$, with Group CNT-Odor-Tastant failing to express an aversion to the LiCl-paired odor cue and Groups PRE-Odor-Odor, PRE-Odor-Tastant, and CNT-Odor-Odor expressing statistically similar aversions in test $(p>.05)$. Notably, previous experience with a tastant prior to odor conditioning facilitated the expression of an aversion to the tastant in test (Group PRE-Odor-Tastant vs. Group CNTOdor-Tastant).

One possible interpretation for conditioning in Group PRE-Odor-Tastant is that conditioning with the odor could have brought forth a representation of the taste

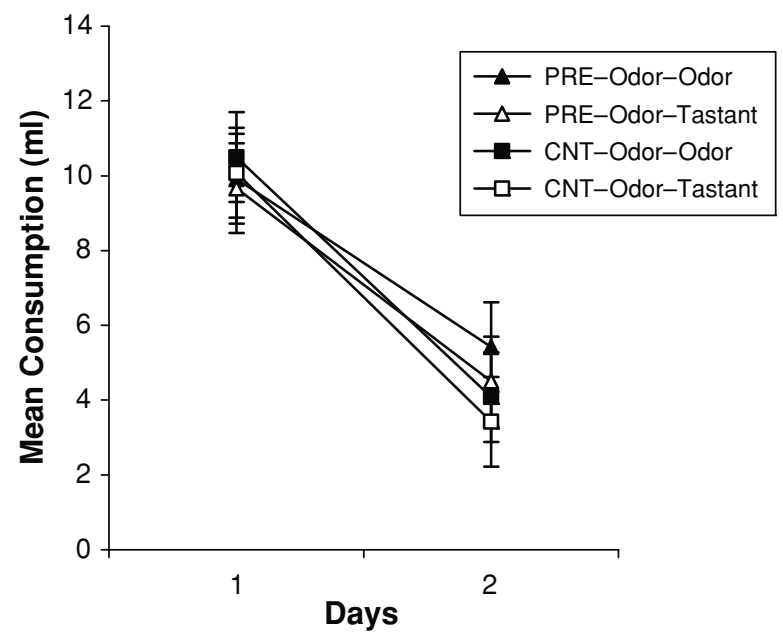

Figure 3. For each group in Experiment 2, mean consumption (in milliliters) during training of the solution that had been paired with $0.15 \mathrm{M} \mathrm{LiCl}$ over days. For clarity, consumption of solutions paired with $0.9 \%$ saline is not included, nor did consumption of these solutions significantly differ over days. Vertical lines represent the standard error of the mean.

component during conditioning, thus allowing the taste component to condition with the odor during training (Holland, 1983; Rescorla, 1981; Rescorla \& Durlach, 1981; Rescorla \& Freberg, 1978). If the taste component conditioned with the odor during training, testing with the tastant should have produced an aversion much stronger than that conditioned to the odor component alone, as was demonstrated in Experiment 1 (Group Tastant-Tastant vs. Group Odor-Odor). In fact, the tastant aversion was only as strong as that conditioned to the odor alone (Group CNT-Odor-Odor). These results suggest that the expression of an aversion to the tastant in Group PRE-OdorTastant was representative of conditioning to the odor, and not to the represented taste component.

Also, if potentiation failed in Experiment 1 because the odor did not generalize to the tastant, we would expect that preexposure to the tastant would facilitate this generalization. In fact, the results did show evidence that preexposure to the tastant facilitates the expression of an aversion to the tastant following odor aversion training. The difficulty with this interpretation, though, is that the subjects preconditioned with a tastant (Groups PRE-Odor-Odor and PRE-Odor-Tastant) in Experiment 2 conditioned the same as Group CNT-Odor-Odor. In all the groups, the expression of an aversion was only as strong as that conditioned to the odor alone. Thus, one may consider that tastant preexposure acted only to reduce neophobic responding to the elements of the tastant. If this were true, we would expect that the strength of an aversion to the odor and tastant would reflect the strength of the odor conditioning in training, which was the result observed in Experiment 2. In addition, if the odor component of the preexposed tastant was detectable during the preexposure phase, this odor preexposure should have caused latent inhibition; in other words, this should have interfered 


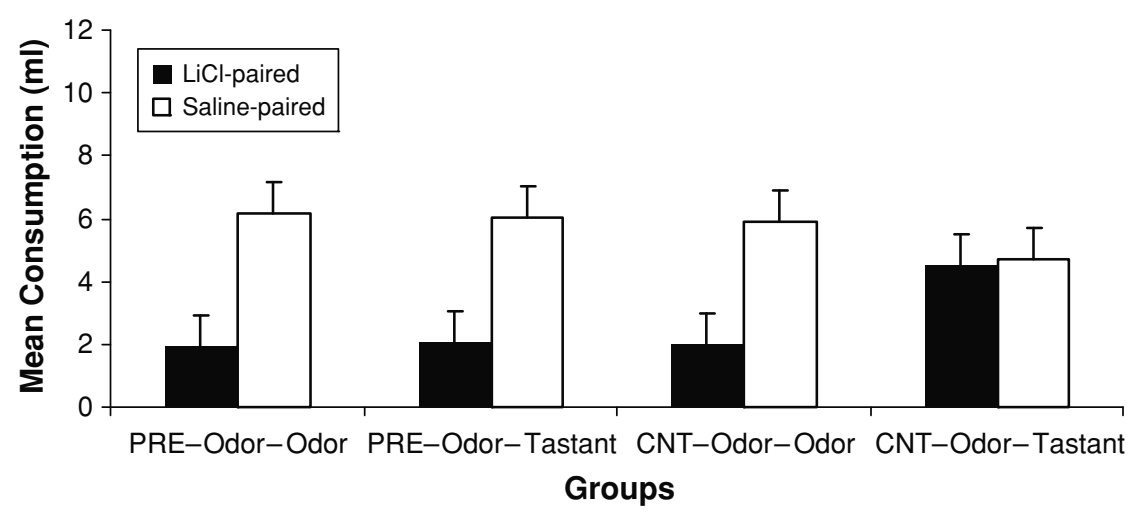

Figure 4. For each group in Experiment 2, mean consumption (in milliliters) pooled across the four test trials of the solution that had been paired with $0.9 \%$ saline versus the solution that had been paired with $0.15 \mathrm{M} \mathrm{LiCl}$ in training. Vertical lines represent the standard error of the mean.

with subsequent odor conditioning in Group PRE-OdorOdor. In fact, there was no evidence of latent inhibition, since the subjects in Group PRE-Odor-Odor expressed aversions of a strength similar to that in a group that did not receive previous tastant experience (i.e., Group CNTOdor-Odor).

One explanation for the apparent absence of latent inhibition is based on the assumption that there is a distinction between the perception of an attribute of a stimulus and the perception of the stimulus itself (Rescorla, 1981; Rescorla \& Freberg, 1978). In the SPC paradigm (AB/B+), where $\mathrm{A}$ is the test cue, B is the trained cue, and " + " denotes an injection of $\mathrm{LiCl}$, it has been argued that when either element (A or B) is presented apart from the compound $\mathrm{AB}$, the organism not only forms a representation of the presented element, but also is able to infer a representation of the nonpresented element (Rescorla, 1981; Rescorla \& Durlach, 1981; Rescorla \& Freberg, 1978). According to this view, subjects perceive the compound $\mathrm{AB}$ as a unitary stimulus, not as an $\mathrm{A} \rightarrow \mathrm{B}$ association. Only with separate element presentations of either A or B will subjects be able to distinguish between the elements of that compound (both the presented and the nonpresented elements). In terms of Experiment 2, the subjects first learned about the odor component alone during training trials (their first experience with separate element presentations with the odor), and not during preconditioning. Thus, tastant preexposure failed to interfere with subsequent odor conditioning due to the lack of an odor representation prior to odor conditioning, which is consistent with the present data.

\section{GENERAL DISCUSSION}

In terms of configural processing (Pearce, 2002), these findings demonstrate that although salt and sweet can be detected by olfaction, this odor has a negligible influence on the strength and effectiveness of conditioning to a tastant. Thus, the generalization decrement from the tastant to the odor is pronounced. Capaldi and colleagues (2004) found that anosmic and intact rats expressed statistically similar aversions to a tastant, suggesting that any ability that the subjects had to smell the solution had no influence on the strength of conditioning to the tastant. Also, Experiment 1 in the present study showed that the strength of the odor aversion following tastant conditioning was not stronger than the strength of the odor aversion following odor-alone conditioning (i.e., Group Tastant-Odor vs. Group Odor-Odor; see Figure 2). These findings suggest that a tastant (i.e., salty and sweet) generalizes almost entirely to its respective "taste" component, and negligibly to its "odor" component when these solutions are presented for ingestion.

Taken together, these findings, combined with those previously reported in Capaldi et al. (2004), strongly support two general conclusions. First, tastant exposure, whether reinforced (Experiment 1) or preexposed (Experiment 2), is both necessary and sufficient for the expression of an aversion to the tastant. Second, the ability of rats to smell these solutions has no measurable effect on the strength of an aversion to a tastant following aversion conditioning to the tastant. Thus, although experimentally naive subjects can smell salt and sweet solutions, conditioned tastant aversion learning is likely not affected by the ability of subjects to smell these stimuli.

\section{REFERENCES}

Benjamin, R. M. (1960). Effect of removal of olfactory bulbs on taste discrimination in normal and brain operated rats. Physiologist, 3, 19.

Brogden, W. J. (1939). Sensory pre-conditioning. Journal of Experimental Psychology, 25, 323-332.

Capaldi, E. D., Hunter, M. J., \& Privitera, G. J. (2004). Odor of taste stimuli in conditioned "taste" aversion learning. Behavioral Neuroscience, 118, 1400-1408.

Domuan, M. (1976). Determinants of the enhancement of flavored-water intake by prior exposure. Journal of Experimental Psychology: Animal Behavior Processes, 2, 17-27.

Ellins, S. R., Cramer, R. E., \& Whitmore, C. (1985). Taste potentiation of auditory aversions in rats (Rattus norvegicus): A case for spatial contiguity. Journal of Comparative Psychology, 99, 108-111.

Ellins, S. R., \& vON KLUge, S. (1987). Preexposure and extinction effects of lithium chloride induced taste-potentiated aversions for spa- 
tially contiguous auditory food cues in rats. Behavioral Neuroscience, 101, 164-169.

HeNKIN, R. I. (1962). Effect of adrenal insufficiency and of corticosteroids on smell threshold. Clinical Research, 10, 400.

Holland, P. C. (1983). Representation-mediated overshadowing and potentiation of conditioned aversions. Journal of Experimental Psychology: Animal Behavior Processes, 9, 1-13.

Pearce, J. M. (2002). Evaluation and development of a connectionist theory of configural learning. Animal Learning \& Behavior, 30, 73-95.

ResCoRla, R. A. (1981). Simultaneous associations. In P. Harzem \& M. D. Zeiler (Eds.), Predictability, correlation, and contiguity (pp. 4780). New York: Wiley.

Rescorla, R. A., \& Durlach, P. J. (1981). Within-event learning in
Pavlovian conditioning. In N. S. Spear \& R. Miller (Eds.), Information processing in animals: Memory mechanisms (pp. 81-112). Hillsdale, NJ: Erlbaum.

Rescorla, R. A., \& Freberg, L. A. (1978). The extinction of withincompound flavor associations. Learning \& Motivation, 9, 411-427.

Rhinehart-Doty, J. A., Schumm, J., Smith, J. C., \& Smith, G. P. (1994). A non-taste cue of sucrose in short-term taste tests in rats. Chemical Senses, 19, 425-431.

Smith, D. V., \& Theodore, R. M. (1984). Conditioned taste aversion: Generalization to taste mixtures. Physiology \& Behavior, 32, 983989.

(Manuscript received December 21, 2005; revision accepted for publication June 20, 2006.) 